OPEN ACCESS

Edited by:

Priscila Casado,

Fluminense Federal University, Brazil

Reviewed by:

Sasanka Chukkapalli,

University of Florida, United States

Alessandro Polizzi,

University of Catania, Italy

*Correspondence:

Pablo Astudillo

pablo.astudillo@uautonoma.cl

${ }^{\dagger}$ Present address:

David González-Quintanilla,

Facultad de Odontología, Universidad

de Chile, Santiago, Chile

Specialty section:

This article was submitted to

Periodontics,

a section of the journal

Frontiers in Dental Medicine

Received: 23 August 2021

Accepted: 29 October 2021

Published: 23 November 2021

Citation:

González-Quintanilla D, Abásolo N and Astudillo P (2021) Wnt Signaling

in Periodontal Disease.

Front. Dent. Med. 2:763308. doi: 10.3389/fdmed.2021.763308

\section{Wnt Signaling in Periodontal Disease}

\author{
David González-Quintanilla ${ }^{\dagger}$, Nicolás Abásolo and Pablo Astudillo* \\ Instituto de Ciencias Biomédicas, Facultad de Ciencias de la Salud, Universidad Autónoma de Chile, Santiago, Chile
}

Periodontitis is a multifactorial and chronic condition associated with the formation of a dysbiotic biofilm, leading to a pro-inflammatory environment that can modulate cell signaling. The Wnt pathway plays fundamental roles during homeostasis and disease, and emerging evidence suggests its involvement in the maintenance of the periodontium and the development of periodontitis. Here, we summarize the role of the $\mathrm{Wnt} / \beta$-catenin and non-canonical Wnt signaling pathways in periodontitis. The accumulated data suggests specific roles for each branch of the Wnt pathway. Wnt5a emerges as a critical player promoting periodontal ligament remodeling and impairing regenerative responses modulated by the Wnt/ $\beta$-catenin pathway, such as alveolar bone formation. Collectively, the evidence suggests that achieving a proper balance between the $\mathrm{Wnt} / \beta$-catenin and non-canonical pathways, rather than their independent modulation, might contribute to controlling the progression and severity of the periodontal disease.

Keywords: Wnt pathway, periodontal disease, periodontitis, Wnt5a, periodontium, inflammation

\section{INTRODUCTION}

Oral diseases constitute a global health problem (1). It is estimated that $99 \%$ of the adult population presents inflammatory gingival disease (2), while the prevalence of severe periodontitis might reach $10-12 \%$ (3). Periodontitis also implies a significant economic burden (4) and is closely related to systemic conditions (5-7). Therefore, we need to better understand the biological basis of periodontitis to address its systemic effects and develop new pharmacological approaches. In this regard, the study of signaling pathways and their modulation during tissue development, maintenance, and disease can contribute to our understanding of periodontitis and provide a source of therapeutic targets.

The Wnt signaling pathway comprises a family of secreted glycoproteins that modulate processes such as embryonic development and cell differentiation, proliferation, survival, and polarity $(8,9)$. Given the multiple biological processes modulated by this pathway, abnormal Wnt signaling has been associated with several diseases, including periodontitis. Consequently, addressing the role of Wnt signaling in this disease might provide new therapeutic approaches and a deeper understanding of the link between periodontitis and other systemic conditions. Therefore, the main goal of this article is to summarize the relationship between periodontitis and the Wnt signaling pathway, extending previous reviews by providing an integrative view encompassing different Wnt signaling modalities, the relative balance between these, and its contribution to specific aspects of periodontal disease. First, a brief overview of this pathway will be provided.

\section{OVERVIEW OF THE WNT SIGNALING PATHWAY}

The Wnt pathway is usually divided among two main branches: the "Wnt/ $\beta$-catenin" (or "canonical") pathway and the "non-canonical" (or "Wnt/ $\beta$-catenin-independent") pathway 
(Figure 1). This pathway requires Wnt ligands, Frizzled (FZD) receptors and a variety of co-receptors (11) and intracellular effectors (Figure 1). Wnt ligands preferentially activate a specific pathway. For instance, Wnt1 and Wnt3a activate the Wnt/Bcatenin pathway, while Wnt5a and Wnt11 activate the noncanonical branch. However, the ability of Wnt ligands to activate a given pathway depends on the availability of specific Frizzled receptors and co-receptors $(12,13)$. For instance, Wnt5a can either activate or inhibit the Wnt/ $\beta$-catenin pathway (14-17). Therefore, assessing the effect of Wnt ligands requires a deep understanding of the cellular context.

The Wnt/ $\beta$-catenin pathway involves the binding of Wnt ligands to FZD receptors and LRP5/6 co-receptors and the subsequent stabilization and nuclear translocation of $\beta$-catenin (Figures 1A,B). Meanwhile, the non-canonical Wnt pathway is mediated by ligands such as Wnt5a and Wnt11, FZD receptors, and co-receptors such as ROR1/2 and RYK, among others (11), triggering intracellular processes that are independent from $\beta$-catenin stabilization (Figure 1C). Importantly, both Wnt branches share some proteins, such as FZD receptors and Dishevelled, leading to potential crosstalk. In addition, several secreted proteins can modulate Wnt signaling. These include Dickkopf (DKK) proteins, soluble Frizzled related proteins (sFRPs), R-spondin proteins, Wnt inhibitory factor 1 (WIF1) (18), and Sclerostin (SOST) (19-21).

As noted above, abnormal Wnt signaling has been related to several diseases. Hyperactivation of the Wnt/ $\beta$-catenin pathway has been linked to cancer (22) and bone pathologies (23), while Wnt5a modulates cell migration and invasion, particularly in the context of metastasis (24), and has also been related to inflammation and fibrosis. For instance, Wnt5a induces the secretion of CCL2/MCP-1, which stimulates the recruitment of monocytes and their maturation to macrophages (25-27).

The Wnt pathway also plays a role in the development and maintenance of periodontal tissues, through the regeneration of both the alveolar bone by modulating the RANK/RANKL/OPG axis (28-30), and the periodontal ligament and cementum (31). Mechanical signals can also modulate Wnt signaling (32), particularly the Wnt/ $\beta$-catenin pathway in the context of bone and teeth (33).

There is a growing body of knowledge associating the Wnt pathways to periodontitis. The evidence discussed in this review suggests that the relative balance between canonical and noncanonical Wnt signaling is lost in periodontitis, implying that restoration of this balance, rather than independent modulation of a specific Wnt pathway, might be required for recovering of periodontal tissues.

\section{WNT SIGNALING AND THE PERIODONTIUM}

Periodontal tissues include the gingiva, periodontal ligament (PDL), alveolar bone, and root cement. The PDL has a rich content of proteins and polysaccharides and is composed of collagen fibers, fibroblasts, epithelial cell rests of Malassez and other cell types $(34,35)$. The functions of the PDL and the remaining support tissues include attaching the tooth to its bony socket, supplying nutrients to the socket and cementum, and protecting the teeth (36). Addressing in full detail the structure of the PDL and its functional relationship with the alveolar bone and cementum is beyond the scope of this article; however, comprehensive reviews have been published recently $(37,38)$.

Gingivitis and periodontitis are inflammatory diseases associated with the formation and persistence of a supra- and sub-gingival bacterial biofilm on the dental surface $(39,40)$. Gingivitis is the first manifestation of the immune response to the biofilm and is characterized by gingival inflammation without loss of periodontal attachment (41). If the biofilm persists, gingivitis becomes a chronic condition that, together with local and systemic risk factors, may favor the progression into periodontitis (42). Periodontitis is characterized by gingival inflammation together with irreversible destruction of periodontal tissues. Importantly, inflammation might lead to altered signaling mechanisms that result in disease pathogenesis (43), linking periodontitis with systemic diseases and promoting the severity of each condition in a bidirectional way, thus strengthening reciprocal oral-systemic associations (5-7).

Importantly, the infection by periodontopathic bacteria and the associated virulence factors and inflammatory mediators, might modulate the Wnt pathway, influencing the progression of periodontitis (44-46). Therefore, it is of great relevance to understanding how the Wnt pathway affects the periodontium during tissue maintenance and disease.

\section{Wnt Signaling in Alveolar Bone and Root Cement Maintenance}

The role of the Wnt signaling pathway in bone metabolism is well-established (47). The Wnt/ $\beta$-catenin pathway is associated with differentiation of mesenchymal stem cells (MSCs) toward the osteoblastic lineage while inhibiting adipogenesis and chondrogenesis $(30,47-49)$. Hyperactivation of the Wnt/Bcatenin pathway leads to an increase in bone volume, while its inhibition correlates with a decrease in bone volume, sometimes with root resorption due to disorganization of PDL collagen fibrils and the absence of cementum (50-52). On the other hand, the non-canonical Wnt pathway might promote osteoclastogenesis (53), although the role of Wnt5a on bone metabolism has not been fully elucidated (54).

The role of the $\mathrm{Wnt} / \beta$-catenin pathway in cementum homeostasis might depend on the level of activation and the cell type (55-59). Negative modulation of the Wnt/ $\beta$-catenin pathway in cells from the PDL might positively modulate the regeneration of cementum $(60,61)$. However, there is increased deposition of cementum in cell lines with higher levels of Wnt/ $\beta$-catenin activity (62). Mechanistically, the Wnt/ $\beta$-catenin pathway induces the expression of Osterix (Osx), leading to DKK1 expression, which dampens Wnt/ $\beta$-catenin signaling (50, 63). Therefore, proper fine-tuning of the $\mathrm{Wnt} / \beta$-catenin pathway might be necessary to promote cementum formation.

Epithelial rests cells from the periodontium, which are sensitive to $\mathrm{Wnt} / \beta$-catenin stimulation, might also be involved in the deposition of cementum during early development (61). 
A

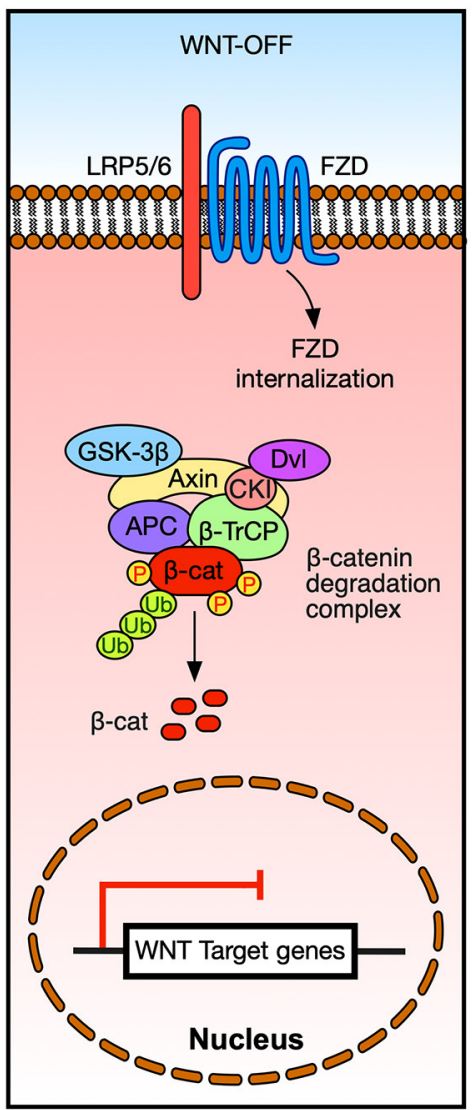

B

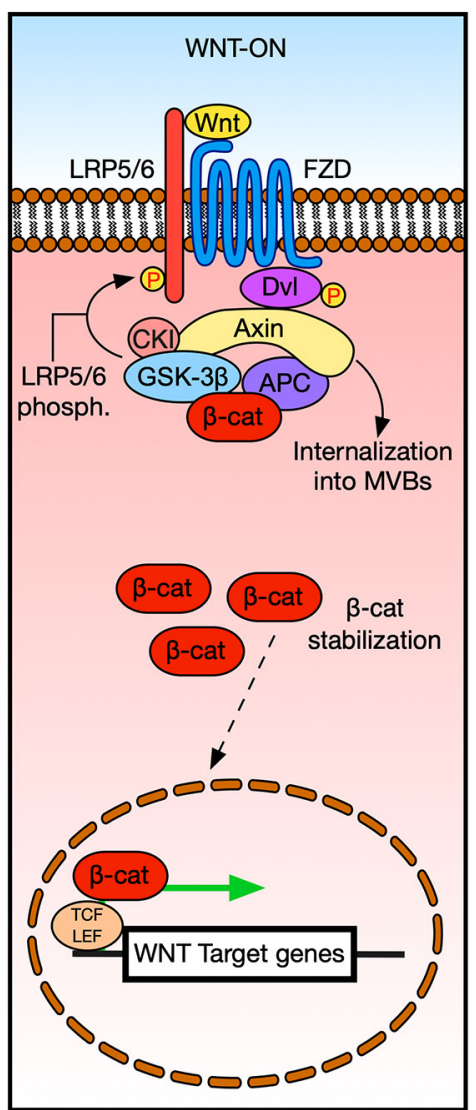

C

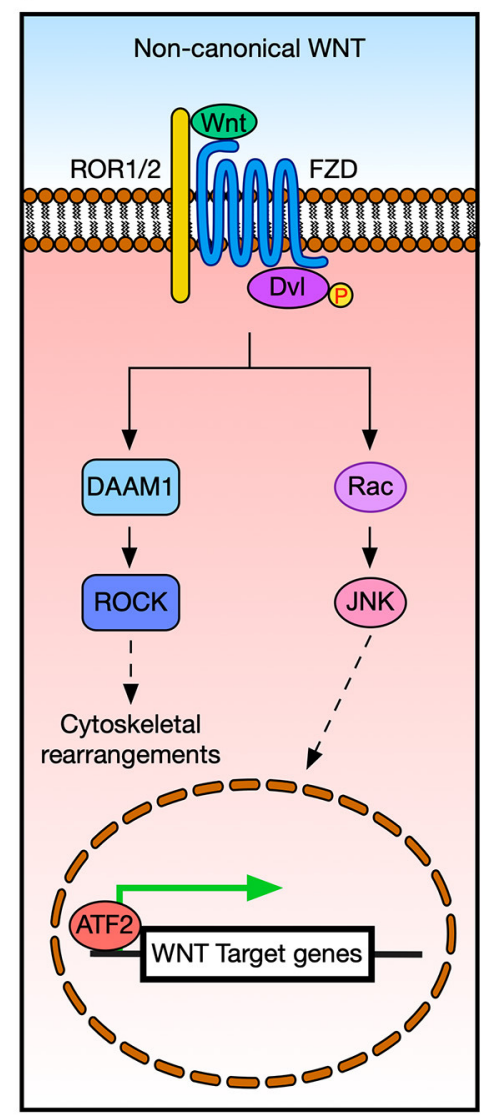

D

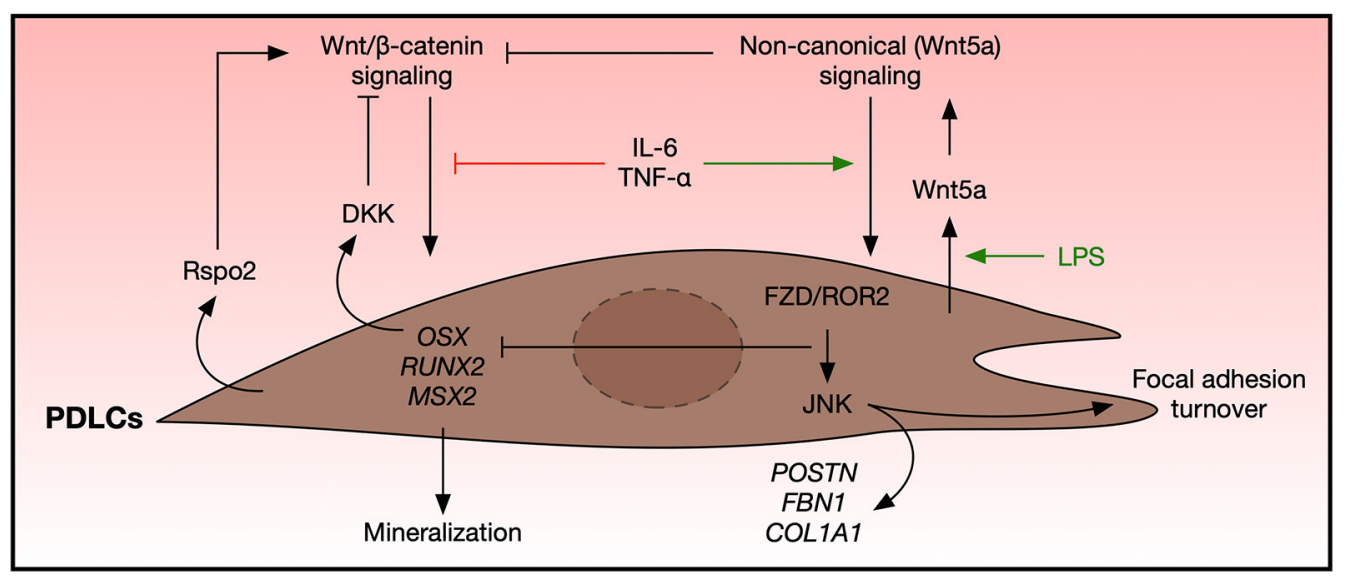

FIGURE 1 | Wnt signaling in the periodontal ligament in homeostasis. (A) Schematic representation of the Wnt/ $\beta$-catenin pathway. In the absence of Wnt ligands, a degradation complex targets $\beta$-catenin for phosphorylation and further degradation. Also, Frizzled (FZD) receptors might be cleared from the plasma membrane by the ZNRF3/RNF43 ubiquitin ligases (not shown). Wnt/ $\beta$-catenin target genes are not expressed in this context. (B) Schematic representation of the Wnt/ $\beta$-catenin pathway in the presence of Wnt ligands. The $\beta$-catenin destruction complex translocates to the plasma membrane in response to early activation events, including LRP5/6 phosphorylation. The destruction complex eventually becomes internalized into multivesicular bodies (MVBs) (10), and newly synthetized $\beta$-catenin becomes stabilized and translocate to the nucleus, where it binds to TCF/LEF transcription factors to promote the expression of target genes. (C) Simplified schematic representation of the non-canonical Wnt pathway. Here, Wht ligands bind to FZD receptors and specific co-receptors, such as ROR1/2 or RYK. Intracellular effectors modulate specific aspects of cell behavior, such as cell migration. Also, ATF2-dependent transcription might be elicited. $\beta$-cat, $\beta$-catenin; WNT-OFF, pathway inactivation in the absence of ligands; WNT-ON, pathway activation in the presence of ligands. For simplicity, some components and receptors are not shown. (D) Cells from the periodontal ligament (PDLCs) are sensitive to different Wnt signals, which in turn promote different responses at the cellular level. Wnt/ $\beta$-catenin signals can promote the expression of osteogenic markers and the secreted inhibitor DKK1, thus modulating the activation of this pathway. On the other hand, non-canonical signaling mediated by Wnt5a might hamper the expression of osteogenic markers while promoting the expression of PDL-related genes and the turnover of cell-matrix adhesion. Additional cues, such as R-spondins, cytokines, or LPS, can modulate the response of PDLCs to specific Wnt inputs. See the main text for details. 
In animal models, it has been observed that Malassez epithelial cells express higher levels of $\mathrm{Wnt} 3 \mathrm{a}$, relative to other cell types from the periodontium (64). In addition, $W n t / \beta$-cateninsensitive stem cells and epithelial cells from the periodontium can differentiate toward a cementogenic lineage to repair root resorption in rats (63).

Altogether, these findings encourage further research regarding the signaling pathways and mechanisms involved in cementum formation and suggest a crucial role for the $\mathrm{Wnt} / \beta$-catenin signaling pathway in this process.

\section{Wnt Signaling and the Periodontal Ligament}

A study employing the murine model of continuous eruption of the incisors described the existence of a gradient of $\mathrm{Wnt} / \beta$ catenin activity across the PDL, with higher activity in the interface between the cementum and the PDL, correlating with higher cell proliferation. In contrast, lower $\mathrm{Wnt} / \beta$-catenin activity is observed in the interface between the PDL and the alveolar bone, matching higher differentiation toward the osteogenic lineage $(51,65)$. This suggests that spatially finetuning of the $\mathrm{Wnt} / \beta$-catenin pathway is necessary for both proliferation and differentiation in the PDL. Later studies using transgenic mice corroborated these findings and demonstrated that excessive activity of the $\mathrm{Wnt} / \beta$-catenin pathway leads to a reduction of the space occupied by the PDL and disorganization of collagen fibers, together with a decrease in bone resorption and alveolar bone accumulation $(50,51)$.

It has also been suggested that human fibroblasts present in the PDL can differentiate toward distinct cell lineages, promoting the nodular formation of mineralized deposits, collagen matrices, and/or reparative fibrous structures in response to periodontal wounds (66). Cells present in the PDL are sensitive to cues that activate the $\mathrm{Wnt} / \beta$-catenin pathway (67), and this pathway might increase PDL mineralization (68). On the other hand, PDL cells cultured in vitro express the mRNAs of WNT5A, ROR2, FZD2, FZD4, and FZD5, and these cells are sensitive to Wnt5a (69). The stimulation of PDL cells with Wnt5a abrogates the expression of osteogenic markers and increases the abundance of POSTN (periostin), FBN1 (fibrillin), and COL1A1. The authors linked these effects to a possible role of Wnt5a in the remodeling of the PDL. Later studies in a cell line derived from immortalized human fibroblasts from the PDL demonstrated that JNK and the non-canonical co-receptor ROR2 are required to mediate Wnt5a signaling (70) (Figure 1D).

Secreted Wnt modulators might also play a role during PDL development and maintenance. Fibroblasts from the PDL express sFRP1, and the inhibition of this protein increased CTNNB1 (the gene encoding for $\beta$-catenin), levels and the expression of osteogenic markers (60). R-spondin proteins are secreted by cells from the healthy PDL in rats and by primary cultures of PDL cells, fulfilling an essential role in regulating the osteoblastic differentiation of immature human PDL cells through the Wnt $/ \beta$ catenin pathway (71). Also, TGF- $\beta$ can activate $\beta$-catenin to modulate the differentiation of PDL cells (72). Finally, an inflammatory context might modulate the Wnt pathway through secreted molecules. For instance, IL- 6 and TNF- $\alpha$ might inhibit the osteogenic and cementogenic differentiation in cells from the PDL by activating the non-canonical Wnt pathway (73) (Figure 1D).

In summary, the cellular components of the periodontium express ligands, inhibitors, and receptors of the Wnt pathway and are sensitive to cues that activate or inhibit this pathway (Figure 2A). Moreover, the evidence obtained from in vitro studies shows that each branch of the Wnt pathway might have different roles: while the $\mathrm{Wnt} / \beta$-catenin pathway has been correlated with the osteogenic differentiation, the non-canonical Wnt pathway might inhibit this differentiation program. Consequently, the study of possible alterations in the Wnt pathway in periodontitis is attractive from a clinical perspective.

\section{WNT SIGNALING IN THE PERIODONTAL DISEASE}

\section{Changes at the Level of Ligands, Receptors, and Modulators of the Wnt Pathway}

Establishing a precise diagnosis for periodontal disease depends on laborious measurements, likely to be influenced by the expertise of the treating physician (74). Therefore, the identification of molecular markers is highly valuable, and several reports have searched for changes in the levels of specific proteins in patients.

One report measured Wnt5a levels in serum, failing to observe differences between subjects with and without periodontitis; however, there was higher expression of sFRP5 in patients without periodontitis, relative to patients with periodontitis followed by tooth loss, leading to changes in the sFRP5/Wnt5a ratio (75). In contrast, two independent studies (44, 76) correlated an increased expression of WNT5A with a higher degree of periodontal destruction and observed lower expression of this ligand in healthy subjects. Also, one study reported higher SOST protein levels in tissue samples from patients with moderate to severe periodontitis, while a similar trend, albeit not statistically significant, was seen for Wnt5a (77). When using a more permissive classification of periodontitis, the difference between disease and control tissues was not observed in samples from gingival crevicular fluid, suggesting that changes in the expression of Wnt5a might be highly restricted to the damaged site in severe periodontitis, thus explaining the absence of significant differences in serum samples (75).

\section{The Wnt Pathway, Dysbiosis, and Inflammation}

In human stem cells from the periodontal ligament cultured on osteogenic medium and stimulated with LPS from E. coli and DKK1, LPS promoted, while DKK1 impaired, osteogenic differentiation (78). Also, proteolysis by gingipains might induce partial degradation of GSK-3 $\beta$, thus increasing the nuclear fraction of $\beta$-catenin (46).

The inflammatory context can also modulate the Wnt pathway. For instance, the treatment of PDL cells with cytokines such as IL- 6 and TNF- $\alpha$ inhibits cell proliferation, the 


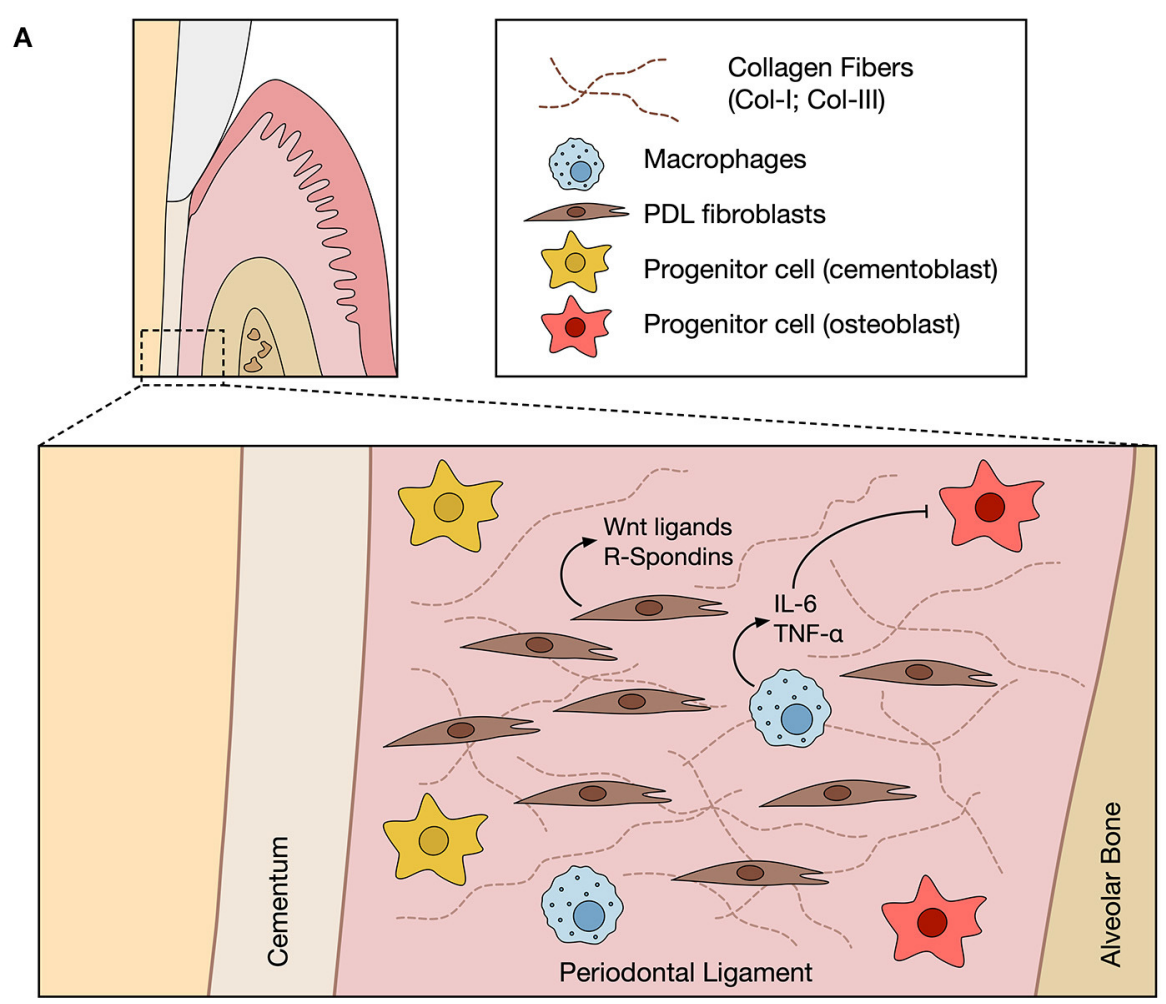

Wnt/ $\beta$-catenin signaling:

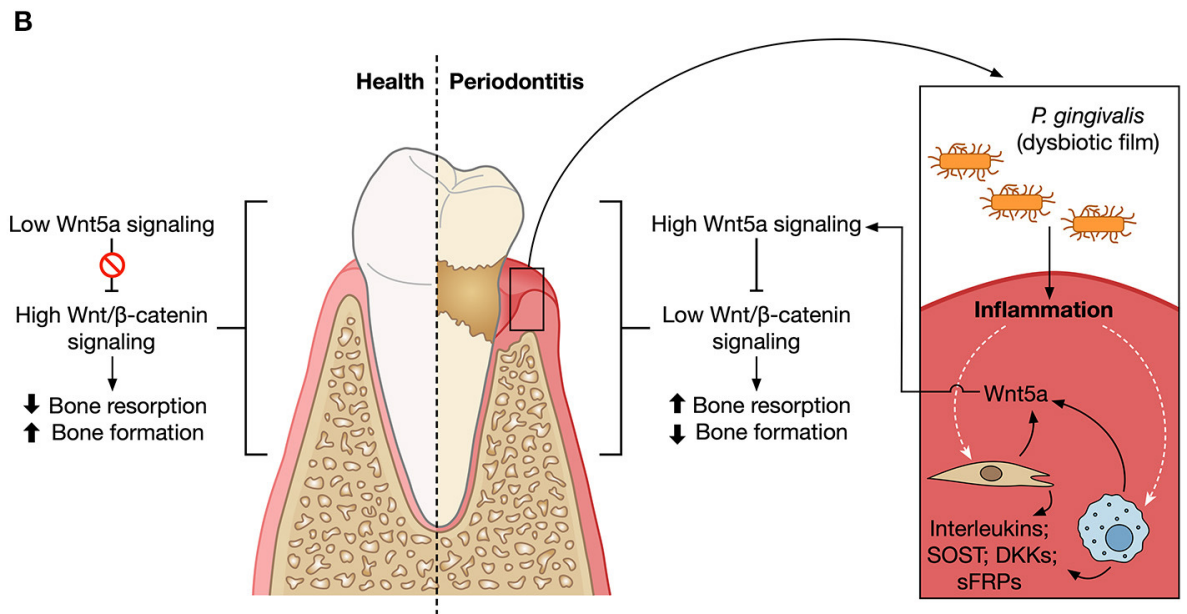

FIGURE 2 | Wnt signaling in periodontium maintenance and periodontitis. (A) The different cellular components of the periodontal ligament (PDL) are embedded in a rich extracellular matrix composed of Collagen I (Col-I) and III (Col-III) fibers. Studies performed in animal models suggest the existence of a gradient of Wnt/ $\beta$-catenin activity across the PDL (black triangle), with higher levels of osteogenic differentiation in areas closer to the alveolar bone. Macrophages within the PDL might secrete factors such as IL-6 or TNF- $\alpha$, which might inhibit the osteogenic differentiation of resident cells in the context of periodontal disease. Secretion of Wnt5a and R-Spondins has also been reported. For simplicity, only the indicated cellular types are shown. (B) In a healthy context, the Wnt/ $\beta$-catenin pathway might have a crucial role in the homeostasis of the PDL and the maintenance of the bone and cementum. However, the challenge mediated by periodontopathic bacteria might promote the local production of Wnt5a, which in turn might contribute to sustaining an inflammatory loop due to its ability to induce the expression of MCP-1, thus promoting the recruitment of macrophages. This can contribute to an imbalance between the Wnt pathway branches, favoring the non-canonical Wnt pathway. Wnt5a might thus promote bone tissue loss, decreasing tooth insertion. P. gingivalis might also induce JAK3 phosphorylation and increased Wnt3a levels (not shown), in turn reducing inflammation. The physiological outcome might thus depend on the relative balance of secreted cytokines and Wnt modulators. Figures based on work created with BioRender.com. 
relative activity of ALP, the expression of genes and proteins related with osteogenic and cementogenic differentiation and the activation of the $W n t / \beta$-catenin pathway (73). More significantly, the same treatments induced an increase in the levels of WNT5A, FZD6, and DKK1, suggesting that the inflammatory context might shift the balance from a Wnt $/ \beta$ catenin to a non-canonical profile, dampening the osteogenic and cementogenic differentiation. In agreement with this notion, blocking the non-canonical Wnt pathway stimulated cementogenesis in these cells, even in the presence of IL- 6 or TNF- $\alpha$ (73).

In addition, the exposure of human gingival cells to LPS from P. gingivalis differentially modulates the expression of WNT5A and SFRP5. While WNT5A levels were increased in subjects with periodontitis and in cells treated with LPS, the levels of SFRP5 were increased in healthy subjects; however, the exposure to LPS induced a reduction in SFRP5 levels (79). LPS from $P$. gingivalis also increases WNT5A levels in the cell line THP1 (44).

Collectively, these observations indicate that the diseased environment shifts the balance between Wnt pathways, favoring Wnt5a-mediated signaling (Figure 2B). Wnt5a has a role in cell migration and invasion, due to its ability to promote both the turnover of focal adhesions (80) and the expression of proteins such as Laminin- $\gamma 2$ (81). Interestingly, the exposure of PDL cells to periodontopathogens and Wnt5a significantly increases proliferation and migration, together with a significant upregulation in the expression of genes such as POSTN, COL1A1, and FBN1 (70). Therefore, the pathogenic challenge in the PDL might lead to changes in the differentiation of progenitor cells and the remodeling of the extracellular matrix through Wnt5a.

MCP-1 might be important during the local inflammatory response by initiating the recruitment of monocytes as a host defense mechanism (82, 83). As noted above, Wnt5a induces MCP-1 expression (25-27). Macrophage infection with $P$. gingivalis increases Wnt5a levels, while the reduction of Wnt5a decreases the production of MCP1 and other pro-inflammatory mediators such as IL- $1 \beta$ and MMP2 (84). In this same study, Wnt5a was shown to be elevated in the gingival tissues of peri-implantitis patients (84).

This evidence suggests a strong link between the noncanonical Wnt pathway and inflammation in periodontitis. However, the Wnt/ $\beta$-catenin pathway might also modulate the inflammatory response. A recent report showed that $P$. gingivalis increases JAK3 phosphorylation in several cell types, including monocytes and innate immune cells; in turn, JAK3 signaling stabilizes Wnt3a, leading to a reduction in pro-inflammatory cytokines, and restricting the severity of periodontitis, since abrogating JAK3 signaling in a mice model of $P$. gingivalis-induced oral infection leads to enhanced bone loss (85). Thus, both Wnt pathways play a role in mediating different aspects of the inflammatory response, further suggesting that proper balance between Wnt modalities might help restoring tissue homeostasis.

\section{Additional Evidence From Experimental Animal Models}

In a murine model of ligature-induced periodontitis (86), local treatment with sFRP5 inhibited inflammation and bone loss, which correlated with a lower number of osteoclasts. Periodontitis was associated with a high expression of Wnt5a and lower expression of sFRP5, a profile that was reversed after sFRP5 treatment (79). Meanwhile, a report employing a rat model of occlusal pressure removal by extraction of opposite teeth showed that Wnt5a levels were decreased in the PDL tissue in sites where the occlusal pressure had been eliminated, relative to controls (69). Therefore, Wnt5a expression is dynamic and can be regulated by either the inflammatory or mechanical context.

On the other hand, in a study using a model of boneperiodontal defect by fenestration in rats, the activation of the $W n t / \beta$-catenin pathway resulted in the significant deposition of cellular cementum and formation of well-organized PDL fibers (68), while another report showed that the constitutive deletion of $D k k 1$ in osteocytes, in the model of ligature-induced periodontitis, abolished alveolar bone loss (87). By histological analysis, the study reported a lower number of inflammatory infiltrates, a reduction in the expression of TNF- $\alpha$ and IL-1 in gingival tissue, and a lower number of osteoclasts, together with an improvement in bone formation.

\section{DISCUSSION}

Collectively, the evidence reviewed in this article shows that the $\mathrm{Wnt} / \beta$-catenin pathway promotes cell proliferation and fine-tuning of this pathway is required for proper osteogenic differentiation. In contrast, the non-canonical Wnt pathway, mainly mediated by Wnt5a, might be hyperactivated in the context of periodontitis, potentially leading to a shift between opposite Wnt modalities. These relationships are summarized in Figure 2.

The role of the Wnt pathway in periodontitis has been reviewed recently $(88,89)$. Here, we complement and advance these observations by showing that proper balance between Wnt modalities, rather than independent modulation of single pathways, might be a better approach to restore tissue balance in periodontitis. Although additional data is required to corroborate this model, the evidence reviewed in this article offers possible avenues to therapeutic approaches. Several modulators of the Wnt pathway have been tested, particularly in the context of cancer research (90). However, drugs targeting specific nodes might be needed to restore the balance between $\mathrm{Wnt} / \beta$-catenin and non-canonical Wnt modalities.

This evidence also unveils open questions. For instance, it will be interesting to determine whether other Wnt ligands are involved in periodontitis progression, or whether other modulators are involved in fine-tuning the activity level of each Wnt pathway, both during tissue maintenance and disease. Also, the precise molecular mechanisms by which periodontopathic bacteria modulate the Wnt pathway remain to be established. 
At the pharmacological level, the identification of specific intracellular transducers of Wnt5a might allow developing targeted drugs to dampen the signaling pathways induced by this ligand. Future studies addressing these and other questions might improve our understanding of periodontitis and provide new therapeutic opportunities.

\section{AUTHOR CONTRIBUTIONS}

DG-Q, NA, and PA contributed to investigation, data interpretation, and drafted the first draft of the article. PA contributed to conceptualization, design, reviewing, and editing of the manuscript. DG-Q and PA contributed to funding

\section{REFERENCES}

1. Petersen PE, Ogawa H. The global burden of periodontal disease: towards integration with chronic disease prevention and control. Periodontol. (2012) 15-39. doi: 10.1111/j.1600-0757.2011.00425.x

2. Farina R, Scapoli C, Carrieri A, Guarnelli ME, Trombelli L. Prevalence of bleeding on probing: a cohort study in a specialist periodontal clinic. Quintessence Int. (2011) 42:57-68.

3. Kassebaum NJ, Bernabé E, Dahiya M, Bhandari B, Murray CJL, Marcenes W. Global burden of severe periodontitis in 19902010: a systematic review and meta-regression. J Dent Res. (2014) 93:1045-53. doi: 10.1177/0022034514552491

4. Listl S, Galloway J, Mossey PA, Marcenes W. Global economic impact of dental diseases. J Dent Res. (2015) 94:1355-61. doi: 10.1177/0022034515602879

5. Hajishengallis G. Periodontitis: from microbial immune subversion to systemic inflammation. Nat Rev Immunol. (2015) 15:3044. doi: $10.1038 /$ nri3785

6. Jepsen S, Caton JG, Albandar JM, Bissada NF, Bouchard P, Cortellini P, et al. Periodontal manifestations of systemic diseases and developmental and acquired conditions: consensus report of workgroup 3 of the 2017 World Workshop on the Classification of Periodontal and PeriImplant Diseases and Conditions. J Periodontol. (2018) 89(Suppl.1):S23748. doi: 10.1002/JPER.17-0733

7. Hajishengallis G, Chavakis T. Local and systemic mechanisms linking periodontal disease and inflammatory comorbidities. Nat Rev Immunol. (2021) 21:426-40. doi: 10.1038/s41577-020-00488-6

8. Logan CY, Nusse R. The Wnt signaling pathway in development and disease. Annu Rev Cell Dev Biol. (2004) 20:781810. doi: 10.1146/annurev.cellbio,.20.010403.113126

9. Willert K, Nusse R. Wnt proteins. Cold Spring Harb Perspect Biol. (2012) 4:a007864-a007864. doi: 10.1101/cshperspect.a007864

10. Taelman VF, Dobrowolski R, Plouhinec J-L, Fuentealba LC, Vorwald PP, Gumper I, et al. Wnt signaling requires sequestration of glycogen synthase kinase 3 inside multivesicular endosomes. Cell. (2010) 143:113648. doi: 10.1016/j.cell.2010.11.034

11. Niehrs C. The complex world of WNT receptor signalling. Nat Rev Mol Cell Biol. (2012) 13:767-79. doi: 10.1038/nrm3470

12. Kikuchi A, Yamamoto H, Kishida S. Multiplicity of the interactions of Wnt proteins and their receptors. Cell Signal. (2007) 19:65971. doi: 10.1016/j.cellsig.2006.11.001

13. van Amerongen R, Mikels A, Nusse R. Alternative Wnt signaling is initiated by distinct receptors. Sci Signal. (2008) 1:re9. doi: 10.1126/scisignal.135re9

14. Torres MA, Yang-Snyder JA, Purcell SM, DeMarais AA, McGrew LL, Moon RT. Activities of the Wnt-1 class of secreted signaling factors are antagonized by the Wnt-5A class and by a dominant negative cadherin in early Xenopus development. J Cell Biol. (1996) 133:1123-37. doi: 10.1083/jcb.13 3.5 .1123 acquisition. All authors contributed to the article and approved the submitted version.

\section{FUNDING}

This work was funded by the following grants: PAI\#77170063 (to Universidad Autónoma de Chile and PA) and Programa de Iniciación Científica, Universidad Autónoma de Chile (to DG-Q).

\section{ACKNOWLEDGMENTS}

The authors thank Dr. Patricio Smith for critical reading of the manuscript.

15. He X, Saint-Jeannet JP, Wang Y, Nathans J, Dawid I, Varmus H. A member of the Frizzled protein family mediating axis induction by Wnt-5A. Science. (1997) 275:1652-4. doi: 10.1126/science.275.5306.1652

16. Mikels AJ, Nusse R. Purified Wnt5a protein activates or inhibits $\beta$ catenin-TCF signaling depending on receptor context. PLoS Biol. (2006) 4:e115. doi: 10.1371/journal.pbio.0040115

17. van Amerongen R, Fuerer C, Mizutani M, Nusse R. Wnt5a can both activate and repress $\mathrm{Wnt} / \beta$-catenin signaling during mouse embryonic development. Dev Biol. (2012) 369:101-14. doi: 10.1016/j.ydbio.2012.06.020

18. Hsieh JC, Kodjabachian L, Rebbert ML, Rattner A, Smallwood PM, Samos $\mathrm{CH}$, et al. A new secreted protein that binds to Wnt proteins and inhibits their activities. Nature. (1999) 398:431-6. doi: 10.1038/18899

19. van Bezooijen RL, Roelen BAJ, Visser A, van der Wee-Pals L, de Wilt E, Karperien M, et al. Sclerostin is an osteocyte-expressed negative regulator of bone formation, but not a classical BMP antagonist. J Exp Med. (2004) 199:805-14. doi: 10.1084/jem.20031454

20. Semënov $M$, Tamai $K$, He $X$. SOST is a ligand for LRP5/LRP6 and a Wnt signaling inhibitor. J Biol Chem. (2005) 280:267705. doi: 10.1074/jbc.M504308200

21. Zhang ZH, Jia XY, Fang JY, Chai H, Huang Q, She C, et al. Reduction of SOST gene promotes bone formation through the Wnt/ $\beta$-catenin signalling pathway and compensates particle-induced osteolysis. J Cell Mol Med. (2020) 24:4233-44. doi: 10.1111/jcmm.15084

22. Anastas JN, Moon RT. WNT signalling pathways as therapeutic targets in cancer. Nat Rev Cancer. (2012) 13:11-26. doi: 10.1038/nrc3419

23. Winkler DG, Sutherland MSK, Ojala E, Turcott E, Geoghegan JC, Shpektor D, et al. Sclerostin inhibition of Wnt-3a-induced C3H10T1/2 cell differentiation is indirect and mediated by bone morphogenetic proteins. J Biol Chem. (2005) 280:2498-502. doi: 10.1074/jbc.M40052 4200

24. Kikuchi A, Yamamoto H, Sato A, Matsumoto S. Wnt5a: its signalling, functions and implication in diseases. Acta Physiol. (2012) 204:1733. doi: 10.1111/j.1748-1716.2011.02294.x

25. Rauner M, Stein N, Winzer M, Goettsch C, Zwerina J, Schett G, et al. WNT5A is induced by inflammatory mediators in bone marrow stromal cells and regulates cytokine and chemokine production. J Bone Miner Res. (2012) 27:575-85. doi: 10.1002/jbmr.1488

26. Jung YS, Lee HY, Kim SD, Park JS, Kim JK, Suh P-G, et al. Wnt5a stimulates chemotactic migration and chemokine production in human neutrophils. Exp Mol Med. (2013) 45:e27. doi: 10.1038/emm.2013.48

27. Li S, Wang W, Zhang N, Ma T, Zhao C. IL-1 $\beta$ mediates MCP1 induction by Wnt5a in gastric cancer cells. BMC Cancer. (2014) 14:480. doi: 10.1186/1471-2407-14-480

28. Kobayashi Y, Thirukonda GJ, Nakamura Y, Koide M, Yamashita $\mathrm{T}$, Uehara S, et al. Wnt16 regulates osteoclast differentiation in conjunction with Wnt5a. Biochem Biophys Res Commun. (2015) 463:1278-83. doi: 10.1016/j.bbrc.2015.06.102 
29. Gibertoni F, Sommer MEL, Esquisatto MAM, Amaral MEC, do Oliveira CA, de Andrade TAM, et al. Evolution of periodontal disease: immune response and RANK/RANKL/OPG system. Braz Dent J. (2017) 28:67987. doi: 10.1590/0103-6440201701407

30. Kovács B, Vajda E, Nagy EE. Regulatory effects and interactions of the Wnt and OPG-RANKL-RANK signaling at the bone-cartilage interface in osteoarthritis. Int J Mol Sci. (2019) 20:184653. doi: 10.3390/ijms20184653

31. Zhao J, Faure L, Adameyko I, Sharpe PT. Stem cell contributions to cementoblast differentiation in healthy periodontal ligament and periodontitis. Stem Cells. (2020) 39:92-102. doi: 10.1002/stem.3288

32. Astudillo P. Extracellular matrix stiffness and $\mathrm{Wnt} / \beta$-catenin signaling in physiology and disease. Biochem Soc Trans. (2020) 48:1187-98. doi: 10.1042/BST20200026

33. Bullock WA, Pavalko FM, Robling AG. Osteocytes and mechanical loading: the Wnt connection. Orthod Craniofac Res 22 Suppl 1. (2019) 1759. doi: 10.1111/ocr.12282

34. Nanci A, Bosshardt DD. Structure of periodontal tissues in health and disease. Periodontol. (2006) 2006:11-28. doi: 10.1111/j.1600-0757.2005.00141.x

35. Chrysanthopoulou A, Mitroulis I, Apostolidou E, Arelaki S, Mikroulis D, Konstantinidis T, et al. Neutrophil extracellular traps promote differentiation and function of fibroblasts. J Pathol. (2014) 233:294-307. doi: 10.1002/path.4359

36. Xiao L, Nasu M. From regenerative dentistry to regenerative medicine: progress, challenges, and potential applications of oral stem cells. Stem Cells Cloning. (2014) 7:89-99. doi: 10.2147/SCCAA.S51009

37. de Jong T, Bakker AD, Everts V, Smit TH. The intricate anatomy of the periodontal ligament and its development: lessons for periodontal regeneration. J Periodontal Res. (2017) 52:965-74. doi: 10.1111/jr e.12477

38. Hirashima S, Kanazawa T, Ohta K, Nakamura K-I. Three-dimensional ultrastructural imaging and quantitative analysis of the periodontal ligament. Anat Sci Int. (2020) 95:1-11. doi: 10.1007/s12565-019-00502-5

39. Mira A, Simon-Soro A, Curtis MA. Role of microbial communities in the pathogenesis of periodontal diseases and caries. J Clin Periodontol. (2017) 44(Suppl.18):S23-38. doi: 10.1111/jcpe.12671

40. Curtis MA, Diaz PI, Van Dyke TE. The role of the microbiota in periodontal disease. Periodontol. (2020) 14-25. doi: 10.1111/prd.12296

41. Mariotti A. Dental plaque-induced gingival diseases. Ann Periodontol. (1999) 4:7-19. doi: 10.1902/annals.1999.4.1.7

42. Schätzle M, Löe H, Bürgin W, Anerud A, Boysen H, Lang NP. Clinical course of chronic periodontitis. I Role of gingivitis. J Clin Periodontol. (2003) 30:887-901. doi: 10.1034/j.1600-051X.2003.00414.x

43. Hasturk H, Kantarci A. Activation and resolution of periodontal inflammation and its systemic impact. Periodontol. (2015) 255-73. doi: 10.1111/prd.12105

44. Nanbara H, Wara-aswapati N, Nagasawa T, Yoshida Y, Yashiro R, Bando Y, et al. Modulation of Wnt5a expression by periodontopathic bacteria. PLOS ONE. (2012) 7:e34434. doi: 10.1371/journal.pone.0034434

45. Yucel-Lindberg T, Båge T. Inflammatory mediators in the pathogenesis of periodontitis. Expert Rev Mol Med. (2013) 15:e7. doi: 10.1017/erm.2013.8

46. Zhou Y, Sztukowska M, Wang Q, Inaba H, Potempa J, Scott DA, et al. Noncanonical activation of $\beta$-catenin by Porphyromonas gingivalis. Infect Immun. (2015) 83:3195-203. doi: 10.1128/IAI.00302-15

47. Baron R, Kneissel M. WNT signaling in bone homeostasis and disease: from human mutations to treatments. Nat Med. (2013) 19:179-92. doi: 10.1038/nm.3074

48. Baron R, Rawadi G, Roman-Roman S. Wnt signaling: a key regulator of bone mass. Curr Top Dev Biol. (2006) 76:10327. doi: 10.1016/S0070-2153(06)76004-5

49. Wang F, Tarkkonen K, Nieminen-Pihala V, Nagano K, Majidi RA, Puolakkainen T, et al. Mesenchymal cell-derived juxtacrine Wntl signaling regulates osteoblast activity and osteoclast differentiation. J Bone Miner Res. (2019) 34:1129-42. doi: 10.1002/jbmr.3680

50. Lim WH, Liu B, Cheng D, Williams BO, Mah SJ, Helms JA. Wnt signaling regulates homeostasis of the periodontal ligament. J Periodontal Res. (2014) 49:751-9. doi: 10.1111/jre.12158

51. Lim WH, Liu B, Mah S, Yin X, Helms JA. Alveolar bone turnover and periodontal ligament width are controlled by Wnt. J Periodontol. (2015) 86:319-26. doi: 10.1902/jop.2014.140286
52. Yin X, Li J, Salmon B, Huang L, Lim WH, Liu B, et al. Wnt signaling and its contribution to craniofacial tissue homeostasis. J Dent Res. (2015) 94:1487-94. doi: 10.1177/0022034515599772

53. Maeda K, Kobayashi Y, Udagawa N, Uehara S, Ishihara A, Mizoguchi $\mathrm{T}$, et al. Wnt5a-Ror2 signaling between osteoblast-lineage cells and osteoclast precursors enhances osteoclastogenesis. Nat Med. (2012) 18:40512. doi: $10.1038 / \mathrm{nm} .2653$

54. Roberts JL, Liu G, Paglia DN, Kinter CW, Fernandes LM, Lorenzo J, et al. Deletion of Wnt5a in osteoclasts results in bone loss through decreased bone formation. Ann N Y Acad Sci. (2020) 1463:45-59. doi: 10.1111/nyas.14293

55. Derubeis AR, Cancedda R. Bone marrow stromal cells (BMSCs) in bone engineering: limitations and recent advances. Ann Biomed Eng. (2004) 32:160-5. doi: 10.1023/B:ABME.0000007800.89194.95

56. Nagatomo K, Komaki M, Sekiya I, Sakaguchi Y, Noguchi K, Oda S, et al. Stem cell properties of human periodontal ligament cells. J Periodontal Res. (2006) 41:303-10. doi: 10.1111/j.1600-0765.2006.00870.x

57. Bae CH, Lee JY, Kim TH, Baek JA, Lee JC, Yang X, et al. Excessive Wnt/ $\beta$ catenin signaling disturbs tooth-root formation. J Periodontal Res. (2013) 48:405-10. doi: 10.1111/jre.12018

58. Cao Z, Liu R, Zhang H, Liao H, Zhang Y, Hinton RJ, et al. Osterix controls cementoblast differentiation through downregulation of Wntsignaling via enhancing DKK1 expression. Int J Biol Sci. (2015) 11:33544. doi: $10.7150 / \mathrm{ijbs} .10874$

59. Choi H, Kim T-H, Yang S, Lee J-C, You H-K, Cho E-S. A reciprocal interaction between $\beta$-catenin and osterix in cementogenesis. Sci Rep. (2017) 7:8160. doi: 10.1038/s41598-017-08607-5

60. Gopinathan G, Foyle D, Luan X, Diekwisch TGH. The Wnt antagonist SFRP1: a key regulator of periodontal mineral homeostasis. Stem Cells Dev. (2019) 28:1004-14. doi: 10.1089/scd.2019.0124

61. Xie X, Wang J, Wang K, Li C, Zhang S, Jing D, et al. Axin2+-mesenchymal PDL cells, instead of K14+ epithelial cells, play a key role in rapid cementum growth. J Dent Res. (2019) 98:1262-70. doi: 10.1177/0022034519871021

62. Wu Y, Yuan X, Perez KC, Hyman S, Wang L, Pellegrini G, et al. Aberrantly elevated Wnt signaling is responsible for cementum overgrowth and dental ankylosis. Bone. (2019) 122:176-83. doi: 10.1016/j.bone.2018.10.023

63. Turkkahraman H, Yuan X, Salmon B, Chen C-H, Brunski JB, Helms JA. Root resorption and ensuing cementum repair by $\mathrm{Wnt} / \beta$-catenin dependent mechanism. Am J Orthod Dentofacial Orthop. (2020) 158:1627. doi: 10.1016/j.ajodo.2019.06.021

64. Nemoto E, Sakisaka Y, Tsuchiya M, Tamura M, Nakamura T, Kanaya S, et al. Wnt3a signaling induces murine dental follicle cells to differentiate into cementoblastic/osteoblastic cells via an osterix-dependent pathway. $J$ Periodontal Res. (2016) 51:164-74. doi: 10.1111/jre.12294

65. Rooker SM, Liu B, Helms JA. Role of Wnt signaling in the biology of the periodontium. Dev Dyn. (2010) 239:140-7. doi: 10.1002/dvdy.22003

66. Smith PC, Martínez C, Martínez J, McCulloch CA. Role of fibroblast populations in periodontal wound healing and tissue remodeling. Front Physiol. (2019) 10:270. doi: 10.3389/fphys.2019.00270

67. Heo JS, Lee S-Y, Lee J-C. Wnt/ $\beta$-catenin signaling enhances osteoblastogenic differentiation from human periodontal ligament fibroblasts. Mol Cells. (2010) 30:449-54. doi: 10.1007/s10059-010-0139-3

68. Han P, Ivanovski S, Crawford R, Xiao Y. Activation of the canonical Wnt signaling pathway induces cementum regeneration. J Bone Miner Res. (2015) 30:1160-74. doi: 10.1002/jbmr.2445

69. Hasegawa D, Wada N, Maeda H, Yoshida S, Mitarai H, Tomokiyo A, et al. Wnt5a induces collagen production by human periodontal ligament cells through TGF $\beta 1$-mediated upregulation of periostin expression. J Cell Physiol. (2015) 230:2647-60. doi: 10.1002/jcp.24950

70. Hasegawa D, Wada N, Yoshida S, Mitarai H, Arima M, Tomokiyo A, et al. Wnt5a suppresses osteoblastic differentiation of human periodontal ligament stem cell-like cells via Ror2/JNK signaling. J Cell Physiol. (2018) 233:175262. doi: 10.1002/jcp.26086

71. Arima M, Hasegawa D, Yoshida S, Mitarai H, Tomokiyo A, Hamano S, et al. R-spondin 2 promotes osteoblastic differentiation of immature human periodontal ligament cells through the $\mathrm{Wnt} / \beta$-catenin signaling pathway. $J$ Periodontal Res. (2019) 54:143-53. doi: 10.1111/jre.12611

72. Lim J-C, Bae SH, Lee G, Ryu CJ, Jang YJ. Activation of $\beta$-catenin by TGF$\beta 1$ promotes ligament-fibroblastic differentiation and inhibits cementoblastic 
differentiation of human periodontal ligament cells. Stem Cells. (2020) 2020:stem.3275. doi: 10.1002/stem.3275

73. Han P, Lloyd T, Chen Z, Xiao Y. Proinflammatory cytokines regulate cementogenic differentiation of periodontal ligament cells by $\mathrm{Wnt} / \mathrm{Ca}(2+)$ signaling pathway. J Interferon Cytokine Res. (2016) 36:328-37. doi: 10.1089/jir.2015.0111

74. Kinane DF, Stathopoulou PG, Papapanou PN. Periodontal diseases. Nat Rev Dis Primers. (2017) 3:17038. doi: 10.1038/nrdp.2017.38

75. Schulz J, Knappe C, Graetz C, Mewes L, Türk K, Black AK, et al. Secreted frizzled-related protein 5 serum levels in human periodontitis-a nested casecontrol study. J Clin Periodontol. (2019) 46:522-8. doi: 10.1111/jcpe.13087

76. Haftcheshmeh SM, Mohammadi A, Soltani A, Momtazi-Borojeni AA, Sattari M. Evaluation of STAT1 and Wnt5a gene expression in gingival tissues of patients with periodontal disease. J Cell Biochem. (2018) 2018:jcb.27487. doi: 10.1002/jcb.27487

77. Chatzopoulos GS, Mansky KC, Lunos S, Costalonga M, Wolff LF. Sclerostin and WNT-5a gingival protein levels in chronic periodontitis and health. $J$ Periodontal Res. (2019) 54:555-65. doi: 10.1111/jre.12659

78. Xing Y, Zhang Y, Jia L, Xu X. Lipopolysaccharide from Escherichia coli stimulates osteogenic differentiation of human periodontal ligament stem cells through Wnt $/ \beta$-catenin-induced TAZ elevation. Mol Oral Microbiol. (2019) 34:12249. doi: 10.1111/omi.12249

79. Maekawa T, Kulwattanaporn P, Hosur K, Domon H, Oda M, Terao Y, et al. Differential expression and roles of secreted frizzled-related protein 5 and the wingless homolog Wnt5a in periodontitis. J Dent Res. (2017) 96:5717. doi: $10.1177 / 0022034516687248$

80. Matsumoto S, Fumoto K, Okamoto T, Kaibuchi K, Kikuchi A. Binding of APC and dishevelled mediates Wnt5a-regulated focal adhesion dynamics in migrating cells. EMBO J. (2010) 29:1192-204. doi: 10.1038/emboj.2 010.26

81. Yamamoto H, Kitadai Y, Oue N, Ohdan H, Yasui W, Kikuchi A. Laminin $\gamma 2$ mediates Wnt5a-induced invasion of gastric cancer cells. Gastroenterology. (2009) 137:242-52.e6. doi: 10.1053/j.gastro.2009.02.003

82. Hanazawa S, Kawata Y, Takeshita A, Kumada H, Okithu M, Tanaka $\mathrm{S}$, et al. Expression of monocyte chemoattractant protein 1 (MCP-1) in adult periodontal disease: increased monocyte chemotactic activity in crevicular fluids and induction of MCP-1 expression in gingival tissues. Infect Immun. (1993) 61:5219-24. doi: 10.1128/iai.61.12.5219-5224. 1993

83. Boström EA, Kindstedt E, Sulniute R, Palmqvist P, Majster M, Holm CK, et al. Increased eotaxin and MCP-1 levels in serum from individuals with periodontitis and in human gingival fibroblasts exposed to pro-inflammatory cytokines. PLoS ONE. (2015) 10:e0134608. doi: 10.1371/journal.pone.013 4608

84. Zhang Q, Liu J, Ma L, Bai N, Xu H. Wnt5a is involved in LOX-1 and TLR4 induced host inflammatory response in peri-implantitis. J Periodontal Res. (2020) 55:199-208. doi: 10.1111/jre.12702

85. Lü L, Yakoumatos L, Ren J, Duan X, Zhou H, Gu Z, et al. JAK3 restrains inflammatory responses and protects against periodontal disease through Wnt3a signaling. FASEB J. (2020) 34:9120-40. doi: 10.1096/fj.201902697RR

86. de Molon RS, Park CH, Jin Q, Sugai J, Cirelli JA. Characterization of ligatureinduced experimental periodontitis. Microsc Res Tech. (2018) 81:141221. doi: $10.1002 /$ jemt.23101

87. Goes P, Dutra C, Lösser L, Hofbauer LC, Rauner M, Thiele S. Loss of Dkk1 in osteocytes mitigates alveolar bone loss in mice with periodontitis. Front Immunol. (2019) 10:2924. doi: 10.3389/fimmu.2019.02924

88. Bao J, Yang Y, Xia M, Sun W, Chen L. Wnt signaling: an attractive target for periodontitis treatment. Biomed Pharmacother. (2021) 133:110935. doi: 10.1016/j.biopha.2020.110935

89. Wei X, Liu Q, Guo S, Wu Y. Role of Wnt5a in periodontal tissue development, maintenance, and periodontitis: implications for periodontal regeneration (Review). Mol Med Rep. (2021) 23:167. doi: 10.3892/mmr.2020.11806

90. Harb J, Lin P-J, Hao J. Recent development of Wnt signaling pathway inhibitors for cancer therapeutics. Curr Oncol Rep. (2019) 21:12. doi: $10.1007 /$ s11912-019-0763-9

Conflict of Interest: The authors declare that the research was conducted in the absence of any commercial or financial relationships that could be construed as a potential conflict of interest.

Publisher's Note: All claims expressed in this article are solely those of the authors and do not necessarily represent those of their affiliated organizations, or those of the publisher, the editors and the reviewers. Any product that may be evaluated in this article, or claim that may be made by its manufacturer, is not guaranteed or endorsed by the publisher.

Copyright (๑) 2021 González-Quintanilla, Abásolo and Astudillo. This is an openaccess article distributed under the terms of the Creative Commons Attribution License (CC BY). The use, distribution or reproduction in other forums is permitted, provided the original author $(s)$ and the copyright owner(s) are credited and that the original publication in this journal is cited, in accordance with accepted academic practice. No use, distribution or reproduction is permitted which does not comply with these terms. 